\title{
Biorobotics: Innovative and Low Cost Technologies for Next Generation Planetary Rovers
}

\author{
Beatrice G. R. Smith, Gregory P. Scott, Dr. Chakravarthini M. Saaj \\ Surrey Space Centre \\ University of Surrey \\ Guildford, Surrey, GU2 7XH, UK \\ ee52bs@surrey.ac.uk, g.scott@surrey.ac.uk, c.saaj@surrey.ac.uk
}

\begin{abstract}
This paper details some of the various robotics projects which have been inspired by the natural world, and which the authors believe will have an impact on the future of robotic space exploration. This includes both hardware-centric projects such as RiSE, and projects which concentrate more on software and control such as Swarm-bots. The authors outline two of the biologically inspired planetary explorer robots currently under investigation at the University of Surrey.
\end{abstract}

Keywords-biomimetics, legged robotics, biorobotics, biological inspiration

\section{INTRODUCTION}

Engineers have been inspired by nature for centuries, from Leonardo da Vinci's flying machines based on birds, to Velcro, based on burrs. Many machines can now move faster than biological systems and can travel through areas too hazardous for living organisms, such as space or high radiation environments. However, machines are not yet as adaptable as organic creatures and it is this adaptability to changing situations which is one of the main focuses of biomimetic engineering.

The following paper reviews some of the ways engineers have taken inspiration from nature. This inspiration is applied to the locomotion systems of many robotic projects, and examples of each are illustrated below. This is followed by an introduction to two new vehicles, each of which biologically inspired in their own respect.

\section{BIOLOGICALLY INSPIRED ROBOTIC EXAMPLES}

\section{A. Scorpion - biologically inspired walking and control}

The Scorpion robot was designed and built at the University of Bremen as a biomimetic eight legged vehicle for moving over rough outdoor terrain [1]. Its main application was intended to be search and rescue; however NASA became interested in the project and is currently testing it as a planetary rover [2].

The Scorpion robot is biologically inspired in both its chassis and its control scheme. It has eight legs, each with three degrees of freedom. The coax joint (closest to the body) moves the leg forwards and backwards, the femur joint raises and lowers the leg, and the tibia joint extends and flexes the leg. The robot can sense the angle and position of each joint, the current drawn by each motor and the load on each foot. This allows it to move over obstacles as high as the robot is long $(650 \mathrm{~mm})$, climb ramps up to $35 \%$, and step over obstacles up to $80 \mathrm{~mm}$ in height. The robot can also adapt the posture of each of its legs, and thus its overall posture. This means that it can raise and lower its body, so that it can move over a wide variety of surfaces without changing its control software.

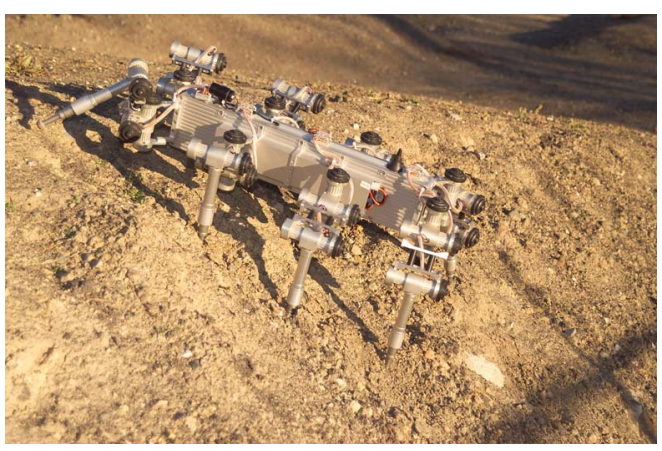

Figure 1. Scorpion under test at NASA Ames. Image taken from [2]

The robot also uses a biomimetic control strategy based on two models: central pattern generators, where the legs are controlled by a central oscillator, and reflexes, where the movement of the actuators is a function of the leg sensor inputs. These models are used instead of conventional linear kinematic models, as the number of actuators and joints in the system means that the linear model would be extremely complex. In contrast, biological systems are easily able to solve problems involving movement of multiple degree of freedom limbs, although they are computationally slower. Both the central pattern generators and reflexes rely on decentralised control, and require little computational power.

Three degree of freedom legs give this robot the ability to accurately place its feet to avoid obstacles. This makes it efficient when moving over rough terrain. Its biologically inspired control system would be especially useful in a space mission as it significantly reduces the need for human interaction. Additionally, its reduced computational power requirements are ideal for low power systems such as those used on planetary rovers.

\section{B. RiSE - a climbing robot}

RiSE (Robots in Scansorial Environments) is a DARPA 
funded project to build a legged robot that can climb buildings [3]. The proposed design is slightly different to most legged robots, as ability to stick to walls is more important than speed. The six legs are therefore underactuated, with two degrees of freedom each. Various innovative MEMS-based feet designs were also investigated to allow the robot to grip onto vertical surfaces.

The chassis design was inspired by a number of principles from nature, including the fact that most animals use a flattened or sprawled posture for climbing, and the fact that many successful climbing animals have a tail or a long body, in order to reduce the force required to pull the animal towards the surface it is climbing. The actual legs of the RiSE robot are fairly basic, having only two joints; the first, nearest the body moves the legs forwards and backwards, while the second moves the leg up and down. However, the legs can move through a much greater angle than many other legged robots. This is because one application of the RiSE robot is climbing trees, and the robot's legs need to be able to reach as far around the tree as possible, to get the best possible grip. The robot also has a third actuator in its legs. This is a spring which extends or contracts the legs, so that it is able to walk with its legs underneath its body, as well as climb with its legs sprawled out. This spring also provides a certain amount of shock absorbency, which improves the robot's climbing ability by minimising the lateral forces, and thus making it harder for the robot to slip.

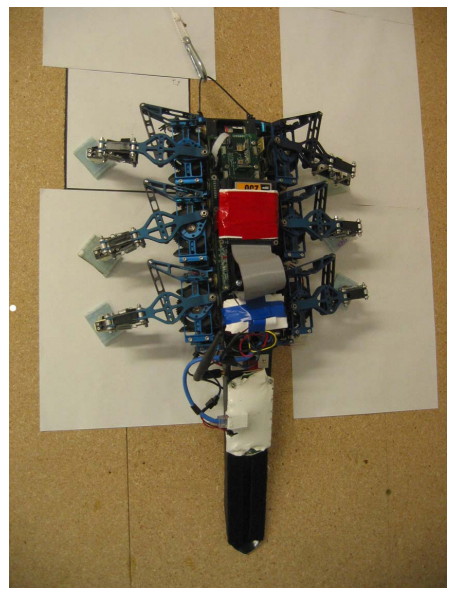

Figure 2. The RiSE robot. Image taken from[4]

Various types of feet were considered for the RiSE robot: Adhesive feet are useful for climbing very smooth surfaces, whereas claws are useful for climbing softer surfaces like bark. For rough but hard surfaces such as brick or concrete, spiny feet are used. These are slightly flexible so as to grip better. RiSE utilises spiny feet based on insect feet. In order for the spines to be small enough to catch on the protrusions of brick and concrete, the feet were made as MEMS components. The robot's ankles were also made compliant so that as much of the foot as possible would be in contact with the surface at any one time.

The RiSE robot uses a modular design; each module is made up of two legs and their control hardware. This is advantageous because it means that experiments can be done in both six and four legged configurations. It also means that there is a certain amount of redundancy; if the controller on one set of legs fails, it will not cause the controllers of the other two sets of legs to fail. The robot has a mass of $3.8 \mathrm{~kg}$, and can carry a payload of $1.5 \mathrm{~kg}$. The fact that this is a climbing robot rather than a walking robot affects its gait; the maximum number of feet must be in contact at all times, and the feet must exert as great a force as possible. This means that a variation on the traditional wave gait is used, with each position of the robot's legs being calculated separately for optimum positioning.

The RiSE robot differs from the other robots in this study in that it is designed for vertical movement, rather than horizontal movement. However, many of its adaptations can be applied to planetary rover design; the sprawled posture and long tail would prevent rovers climbing steep slopes from toppling over backwards, the compliant legs would improve movement over rough terrain, and reduce falling damage, and the MEMS feet could be designed in various ways depending on the application the rover is designed for. A climbing rover such as this could be useful for exploring areas such as the walls of the canyons of Mars, which are inaccessible to the current generation of rovers, or it could be adapted to stick to the outside of space craft to help astronauts with extra vehicular activities.

\section{Lobster - novel actuators for underwater robotics}

The DARPA lobster robot program (or Biomimetic Underwater Robot Program) focused on building a robot that could move about on the seabed, searching for mines and disarming them [5]. This is an application which is ideal for robots, as it would be extremely dangerous for humans. It was found that lobsters and crabs had evolved to move easily about the seabed, even when there are strong currents or turbulence. They also have a neurological system which can be translated into hardware, which makes it useful for controlling a robot. A lobster's shape is hydrodynamically efficient and it has the ability to walk backwards and sideways, as well as forwards. The neurological systems of lobsters are based around a combination of walking and floating behaviours to hunt for food, adapting their movement based on their environment. This is an ideal basis for a control system to hunt for mines in a changing underwater environment. For these primary reasons, the robot was based on a lobster. 


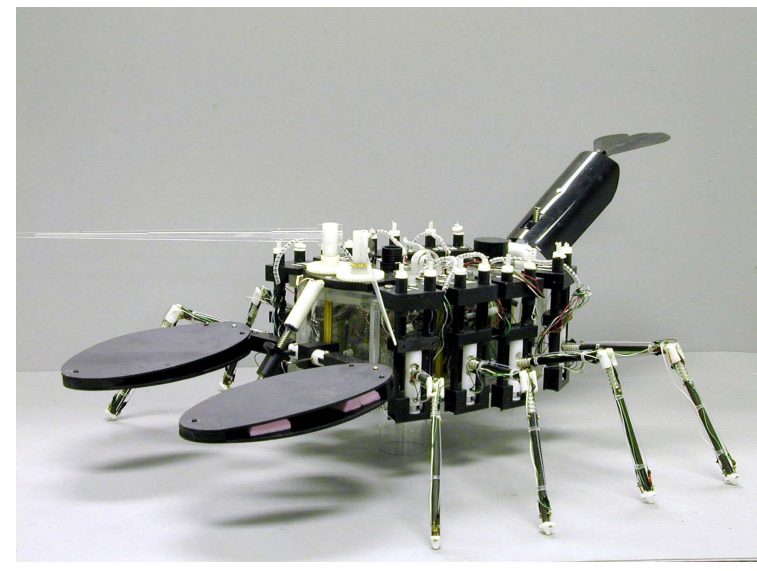

Figure 3. The Lobster Robot. Image taken from [6]

The control of the lobster robot is based on the command neuron, coordinating neuron, central pattern generator (CCCPG) model, similar to that used in the Scorpion robot. It is implemented using a finite state machine made up of three major components; the oscillator which regulates the period of the leg movements, the co-ordinator which controls which combinations of legs move, and the recruiter which monitors and controls the loads on the legs [7].

The legs of the lobster robot are actuated using Nitinol, a shape memory alloy consisting of nickel and titanium [8]. Shape memory alloys (SMA) are metals which change their structure when heated, thus contracting. Nitinol is used as, of the various alloys available, its stress strain characteristics are closest to crustacean muscles, and would thus work well with the CCCPG control system. It is also very lightweight, which is an advantage in a robot that floats as one method of locomotion. In order to increase the load capability, bundles of Nitinol wires are used, in a similar way to bundles of muscles in a biological system. The robot has eight legs, with three degrees of freedom each. In addition to moving forwards and backwards, up and down, the robot's legs can also extend and flex, so that it can move sideways. Each joint is actuated using antagonistic pairs of Nitinol wire bundles that can be actuated in a graded fashion, so as to fix the joint angles to specified points. These are activated using pulse width modulation so as to prevent the Nitinol over heating and burning out. Power consumption is minimised by insulating the Nitinol with PTFE tubing. While the Nitinol reduced mass, it is difficult to control, and a lot of power is required for a large robot.

\section{Swarm robots - distributed intelligence based on insect colonies}

The Swarm-bot was a 42 month project started in March 2001 by a number of universities in Europe sponsored by the European Community Future and Emerging Technologies program [8]. The aim of the project was to design and implement a single robot, called a Swarm-bot, made up of 1030 smaller robots which were called S-bots. This differs from previous projects concerning reconfigurable robots as a distributed adaptive control architecture is used, rather than a centralised one. This means that each S-bot can operate on its own, as well as co-operating with the rest.
This concept was inspired by colonies of insects such as bees or ants, which work together to achieve goals which a single insect could not, for example some species of ants link themselves together to form a bridge over large gaps, allowing other ants to pass over it. Swarm robotics could be applied to a number of areas, such as search and rescue or the exploration of space and other extreme environments.

Each S-bot has a circular chassis with a $116 \mathrm{~mm}$ diameter. They use a track/wheel hybrid locomotion system which allows them to move over moderately rough terrain. Each robot had a pole which can be lifted and lowered that carries a camera for navigation and can also be used to right the robot if it capsized. Each S-bot can connect itself to other S-bots via a rigid gripper, or via two semi flexible velcro connectors. The rigid gripper is powerful enough to lift another S-bot if required. The robots communicate between each other via a ring of LEDs around the circumference of the chassis. These LEDs can flash in varying colours and frequencies, allowing the robots to send messages.

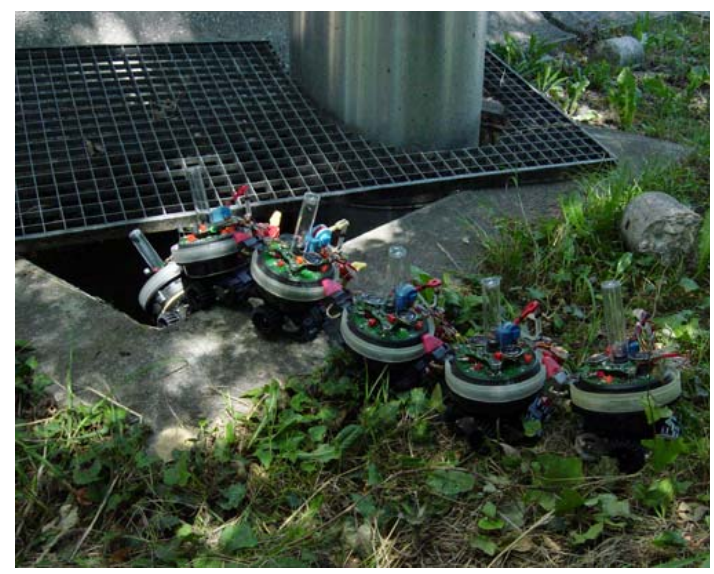

Figure 4. A Swarm-bot made up of six S-bots. Image taken from [9]

Originally each robot was programmed with a number of pre designed behaviours depending on the scenario to be tested. This was later extended to adaptive neural networks which enable the S-bots to devise their own communication systems based on their ability to detect light and sound [10]. The S-bots also use evolutionary algorithms to decide whether they can complete a task on their own. If not, they can assemble with other S-bots to complete the objective [11].

The Swarm-bot represents one of the key factors described in the introduction to this study - adaptability. A swarm of small robots could spread out to investigate a large area in less time than a conventional sized rover, but if an impassable obstacle such as a large cliff or trench is encountered they could work together to get over it. Evolutionary algorithms and machine learning would mean even less human interaction is required, as well as making the swarm more robust. A further advantage of using a swarm is that one or more robots can be lost or destroyed without having too great an impact on the swarm as a whole, as long as the original number of S-Bots is high enough. This redundancy would make the swarm as a whole more reliable and able to work for longer.

The main problems with using a swarm of robots in space 
would be that in order to minimise mass and space very small nanorovers (i.e. rovers with a mass less than $1 \mathrm{~kg}$ ) should be used. Ensuring these nanorovers can carry a useful payload and have a solar panel area large enough to power the rover, while maintaining the small size would be challenging. Similar research is being conducted at the Surrey Space Centre on nano-satellite swarms, the technology from which can similarly be applied to ground-based planetary rovers [12].

\section{INTRODUCING TWO NOVEL BIOLOGICALLY INSPIRED ROBOTS}

\section{A. SMA Actuated Nanorover}

When planning a planetary rover mission, size and mass are important considerations. The larger and heavier the rover is, the higher the cost (both financially and regarding mission design constraints) to get the rover to its destination, and power itself on the surface. For this reason the Surrey Space Centre is proposing a nanorover, with a mass less than $1 \mathrm{~kg}$, which would nevertheless have a greater trafficability than the $11.5 \mathrm{~kg}$ Sojourner microrover.

In order to maximise the manoeuvrability and agility of the nanorover, a legged design was chosen since this would offer better all terrain performance than a wheeled rover, but would not result in the increase in the mass and power requirements often associated with tracked vehicles. A legged vehicle could also adapt more easily to the environment by changing its stance to move between gaps or sprawling to climb steep slopes or even cliffs.

\section{1) System overview}

The robot was designed to be as small as possible while still being big enough to carry not only the payload, but also a solar panel sufficient to power the rover and its payload, the middle legs are offset from the central axis of the rover to improve stability and reduce the forces required to support the robot [13]. The legs themselves have only 2 degrees of freedom. This is to reduce the complexity and power requirements of the rover. In order to reduce mass carbon fibre was selected for the legs and chassis.

The forward (thorax) and rear (abdomen) sections of the robot have a passive vertical coupling between them. This mimics the joint in an insect's body between the thorax and abdomen. This will work in a similar way to the rocker bogie system seen on wheeled rovers such as Sojourner and the MERs to improve the ability of the rover to surmount obstacles. The thorax can be lifted onto an obstacle by the front legs, while the rear legs remain on the ground. This improves the stability and reduces the likelihood of slippage.

In order to improve stability when climbing slopes or over obstacles the robot has a 'tail', which is held against the ground with a spring. This will assist the rover from tipping over backwards when on an incline. The tail could also be actuated to be used as a self righting mechanism; if the robot falls on its side, it could push itself back onto its feet using the tail. A further adaptation is the inclusion of springs in the leg joints to give some compliancy and thus improve performance on uneven surfaces, as the leg angles will be passively adjusted to give the maximum amount of ground contact.

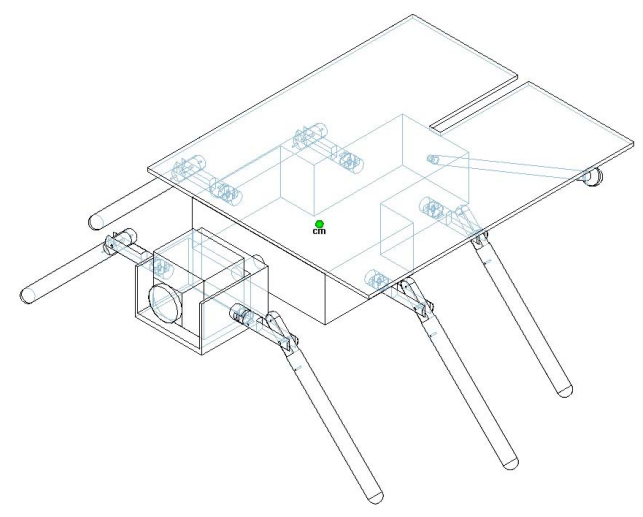

Figure 5. Initial design of nanorover

\section{2) Actuation}

Although the high power requirement and the difficulty of control are problems for a planetary rover, SMAs offer intriguing possibilities for space robotics due to its small size and high force to mass ratio. Since the power required increases proportionally to the length of SMA a smaller robot with smaller actuators will have lower power consumption, making it more suitable for nanorovers than larger vehicles. SMA actuators have already been used in space as part of an experiment on Sojourner to measure dust build up, however there has been little work done on the effects of the space environment on the material. It has been found that SMA actuators only contract by a small fraction of their total length, so the legs were designed to be a lever with a 1:6 ratio, allowing the rover to lift its legs further. This also allows for a shorter SMA and thus requiring less power for actuation. The leg design is shown in Error! Reference source not found.

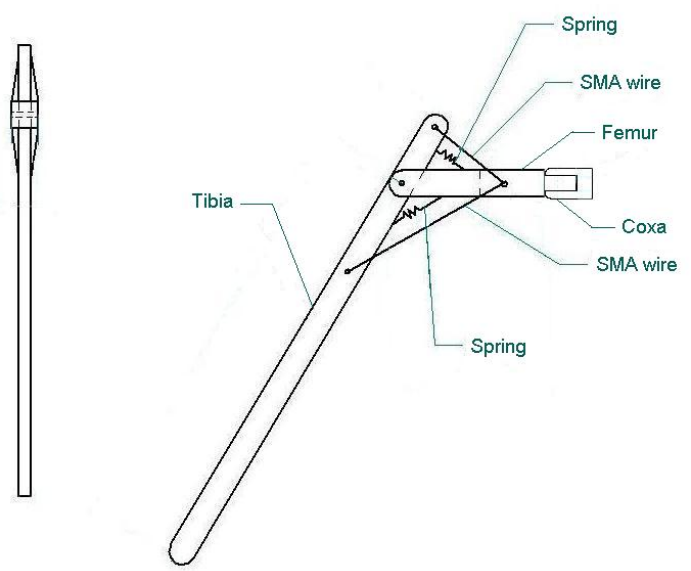

Figure 6. Leg design showing placement of SMA

\section{3) Summary}

The main focus of this robot design is on minimising size and mass, while maintaining operational capabilities. Various design features were inspired by insects, including the six legs with compliant joints and segmented body. The rover is also actuated using shape memory alloy actuators, which simulate biological muscle and allows further reductions to the vehicle size and mass. This rover would be ideal for use as part of a swarm due to its low mass and size. 


\section{B. CAPTAIN microrover}

The Carausius morosus All-Purpose Terrain-Autonomous Insect Navigator (CAPTAIN) is a biologically inspired areonaut assistant robot this is proposed by the Surrey Space Centre. This legged vehicle is directly inspired from the Carausius morosus, more commonly known as the stick insect or walking stick. This vehicle can be utilised by areonauts for a variety of tasks from a scientific assistant carrying experimental payloads to a "pack mule" type assistant for carrying equipment over difficult terrain. Alternatively is can be utilised autonomously without direct human interaction. A generalised concept of the vehicle is shown in Figure 7. Figure 7.

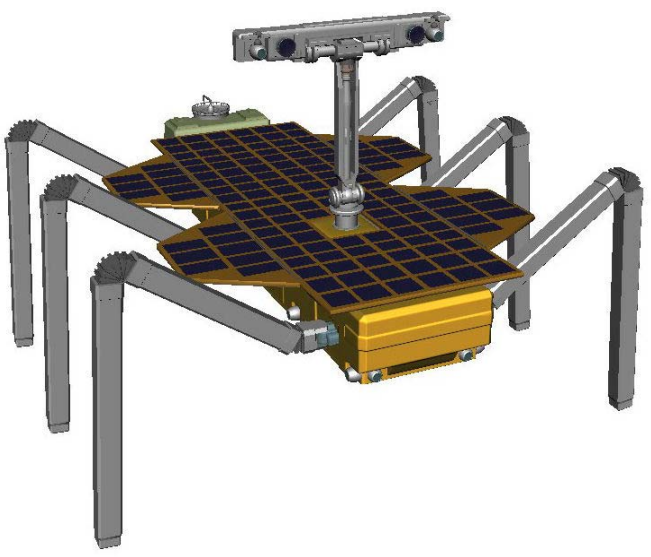

Figure 7. Design concept for the CAPTAIN rover. Image courtesy: B. Otenio.

An insect locomotion baseline was selected, similarly to those discussed in Section II, due to the primary concern over the ability of traditional rover designs (i.e., wheeled or tracked) to traverse the less open terrain of some areas of the Martian surface. As described aboveII, a biologically inspired legged vehicle will have the capability to traverse more difficult terrain than the traditional wheeled or tracked counterparts of similar size [14].

\section{1) System Overview}

CAPTAIN is powered by batteries that are recharged by solar panels on the top surface of the vehicle. These panels were sized based on motor torque requirements for walking and the additional power required to run core processes while walking, as this is the most power-intensive period for the vehicle. They will unfold outward and to the sides of the vehicle above the spaces between each leg, as this will not interfere with locomotion and provide additional surface area for solar cells to generate power.

The vehicle legs, stereoscopic camera system and solar panels can be folded during transit to Mars as shown in Figure 8. Figure 8. Upon landing the front solar panels deploy to generate power for the batteries. Then the stereoscopic camera boom is extended to allow the rear solar panels to unfold. Finally, when enough power is generated, the legs can be extended.

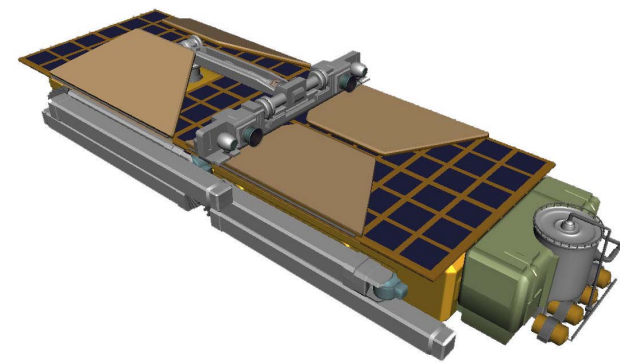

Figure 8. CAPTAIN rover in stowed position before deployment. Image courtesy: B. Otenio.

Various scientific payloads can be used with this system. Figure 8. Figure 8. shows a generic payload with a cylindrical collection subsystem for easy access to an areonaut, as needed. The system is designed for convenient access to suited areonauts and hot-swappability with other desired scientific payloads.

\section{2) Mobility system}

The vehicle mobility system was inspired by the stick insect in both segment dimensioning and joint movement capabilities. The leg segment lengths were designed to be near proportional to the body length, though the robot's leg segments are slightly shorter in proportion to help reduce motor torque requirements for each stancing leg.

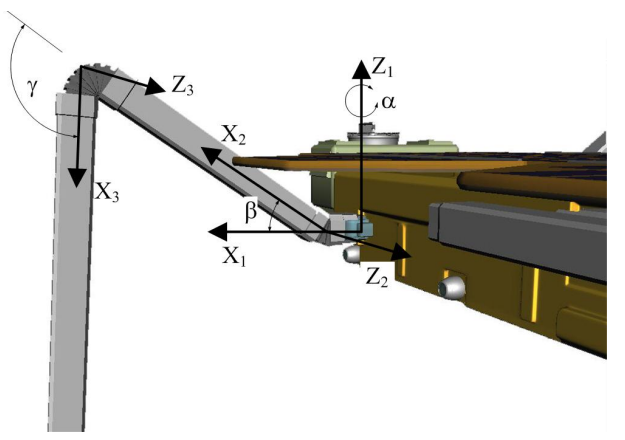

Figure 9. The back-right leg segment of CAPTAIN, labelled with joint axes and range of motion. Image courtesy: B. Otenio.

The legs are $4 \mathrm{~cm}$ by $4 \mathrm{~cm}$ rectangular aluminium tubing with a $2 \mathrm{~mm}$ wall thickness and segment lengths as noted in Table 1 and shown graphically in Figure 9. Rectangular tubing was selected instead of cylindrical because modelling the mechanical response of the soil is simplified when using flat plates [15], and therefore the vehicle's tractive capability can be more accurately determined.

TABLE I. LEG SEGMENT DETAILS FOR CAPTAIN ROVER

\begin{tabular}{|c|c|c|c|}
\hline Segment & $\begin{array}{c}\text { Coxa } \\
\text { (1) }\end{array}$ & $\begin{array}{c}\text { Femur } \\
\text { (2) }\end{array}$ & $\begin{array}{c}\text { Tibia } \\
\text { (3) }\end{array}$ \\
\hline $\begin{array}{c}\text { Segment Length } \\
{[\mathrm{m}]}\end{array}$ & 0.05 & 0.31 & 0.47 \\
\hline $\begin{array}{c}\text { Segment Mass } \\
\text { (w/motors) [kg] }\end{array}$ & 0.378 & 0.545 & 0.648 \\
\hline $\begin{array}{c}\text { Joint Direction } \\
\text { (wrt body) }\end{array}$ & front/back & up/down & up/down \\
\hline $\begin{array}{c}\text { Range of Motion } \\
{\left[{ }^{\circ}\right]}\end{array}$ & $\alpha=80^{\circ}$ & $\beta=60^{\circ}$ & $\gamma=100^{\circ}$ \\
\hline \multicolumn{3}{|c|}{}
\end{tabular}


3)

Vehicle control

This vehicle is utilises a multi-tiered control system with separate controllers for navigation, gait, leg and joint control. Although each is based loosely on the stick insect, simplification must be made for onboard processing, etc. One bio-inspired control capability that is considered is the utilisation of the Winters and Stark muscle model within the joint controller [16]. Additionally, leg control must consider the vehicle's sinkage into soil with each step, as well as the expected slip the vehicle will encounter while walking [17].

In summary, this vehicle is biologically inspired in a number of ways. Primarily the entire mobility system mimics the stick insect with regards to leg placement, segment dimensions and joint capability. Additionally, the controller is inspired biologically with visco-elastic muscle modelling considered for each joint and soil strength considered with each step.

\section{CONCLUSIONS:}

A wide variety of biologically inspired advances have been made in robotics, many of which can be applied to planetary exploration. The legged vehicle offers the advantages of agility and adaptability over a wheeled or tracked vehicle, while control algorithms based on biological systems such as CCCPG models, evolutionary algorithms and neural networks go some way to reduce the inherent complexity of controlling a legged vehicle. New advances in materials and manufacturing technologies, such as MEMS and shape memory alloy and carbon nanotube muscles will allow engineers to design rovers which are smaller and lighter, which could even be used in swarms to explore new worlds.

Two new examples of biologically inspired planetary rovers are also proposed. The first is a nanorover using shape memory alloy actuators in order to reduce vehicle size and mass. The second is a larger microrover designed to act autonomously or assist areonauts in their scientific investigations on the surface of Mars.

\section{V.REFERENCES}

[1] Spenneburg, D., "Scorpion - outdoor-capable walking robot"

[2] John Bluck "NASA evaluates eight-legged Scorpion robot for future exploration" Online article available at http://www.nasa.gov/centers/ames/research/exploringtheuniverse/scorpio $\mathrm{n}$ robot.html

[3] Spenko, M. J., Haynes, G. C., Saunders, J. A., Cutkosky, M. R., Rizzi, A. A., Full, R. J. and Koditschek, D. E., "Biologically inspired climbing with a Hexapedal Robot", Journal of Field Robotics 25(4-5), 223-242 (2008)

[4] D. Pescovitz, “"Robobugs, Gecko Tape, and Nature's Inspiration" In: ScienceMatters@Berkeley, Volume 3, Issue 19 March 2006

[5] Kirchner, F., Spenneberg, D., and Linnemann, R., "A Biologically Inspired Approach Toward Robust Real-World Locomotion in Legged Robots". Neurotechnology for Biomimetic Robots, MIT Press, Cambridge, MA, USA, 2002, pp. 419-448.

[6] J.Ayers "Biomimetic Underwater Robot Program" Online at http://www.neurotechnology.neu.edu/

[7] Ayers, J. (1995) "A Reactive Ambulatory Robot Architecture for Operation in Current and Surge.” In: Proc. of the Autonomous Vehicles in Mine Countermeasures Symposium. Naval Postgraduate School. Pp. $15-31$

[8] Witting, J., Koray, S. and Adams, G. G., "SMA Actuators Applied to Biomimetic Underwater Robots"

[9] M. Dorigo, "Swarm-bots: Swarms of self-assembling artefacts" Online at http://www.swarm-bots.org/

[10] Dorigo M. "SWARM-BOT: An experiment in swarm robotics" In P. Arabshahi and A. Martinoli, editors, Proceedings of SIS 2005 -- 2005 IEEE Swarm Intelligence Symposium, pages 192-200, IEEE Press, Piscataway, NJ, 2005

[11] Ampatzis C., Tuci E., Trianni V. and Dorigo M. "Evolution of Signalling in a Group of Robots Controlled by Dynamic Neural Networks" In E. Sahin et al., Proceedings of the Second Workshop on Swarm Robotics, In press.

[12] Tuci E., Groß R., Trianni V., Mondada F., Bonani M. and Dorigo M "Cooperation through self-assembly in multi-robot systems" ACM TAAS, Volume 1(2), 115-150, December 2006

[13] Vladimirova, T., Wu, X., Bridges, C. P. "Development of a Satellite Sensor Network for Future Space Missions." Proceedings of the IEEE Aerospace Conference 2008. 01-08 March 2008.

[14] Gonzalez de Santos, P., Estremera, J., Garcia, E., "Optimising Leg Distribution Around the Body in Walking Robots." Proceedings of the 2005 IEEE International Conference on Robotics and Automation Barcelona, Spain, April 2005

[15] Ellery, A., Scott, G. P., Husbands, P., Gao, Y. et. al., (2005). "Bionics \& Space Systems Design Case Study 1 - Mars Walker," Report Number: Contract AO/1-4469/03/NL/SFe, European Space Agency, Advanced Concepts Team.

[16] Scott, G. P., M. C. Saaj, E. Moxey (2008). Walking Microrovers for Planetary Exploration: Investigation into the Mechanics of SoilFootprint Interaction. International Society of Terrain Vehicles Systems (ISTVS) 2008 Conference. Turin, Italy. 06-09 November 2008.

[17] Winters, J. M. and Stark, L., "Analysis of Fundamental Human Movement Patterns Through the Use of In-Depth Antagonistic Muscle Models.," IEEE Transactions on Biomedical Engineering,, Vol. 32, No. 10,1985 , pp. 826-837.

[18] Scott, G. P., Saaj, C. M., and Moxey, E., "Modelling Soil Traction for more Effective Control of Walking Planetary Rovers," Proceedings of the Towards Autonomous Robotics Symposium (TAROS), 2008. 